\title{
ROTATING ORBITS OF PENDULUM IN STOCHASTIC EXCITATION
}

\author{
Sze-Hong Teh, Ko-Choong Woo, Hazem Demrdash \\ University of Nottingham Malaysia Campus, Department of Mechanical, Materials and Manufacturing Engineering, \\ Semenyih, Malaysia \\ e-mail:kedx1tsn@nottingham.edu.my; Woo.Ko-Choong@nottingham.edu.my; Hazem.Demrdash@nottingham.edu.my
}

\begin{abstract}
A method to extract energy from an excitation which is stochastic in nature is presented. The experimental rig comprises a pendulum, and a vertical excitation is provided by a solenoid. The control input assumed in the form of a direct current motor, and another motor, used in reverse, acts as a generator. The stochastic excitation has been achieved by varying the time interval between switching the RLC circuit on and off according to a random distribution. Such non-linear vertical excitations act on an oscillatory system from which a pendulum is pivoted. The Pierson-Moskowitz spectrum has been chosen as the random distribution while an inverse transform technique has been used for generation of the random excitation signal in LabVIEW environment. Moreover, a bang-bang control algorithm has been implemented to facilitate rotational motion of the pendulum. Experimental observations have been made for various noise levels of vertical excitations, and their implication on energy generation has been discussed. A positive amount of energy has been extracted for a minimal amount of the control input.
\end{abstract}

Keywords: parametric pendulum, stochastic excitation, energy harvesting

\section{Introduction}

Energy in form of mechanical vibration generally exists everywhere, all the time, and its source can be natural (e.g. sea wave) or artificial (e.g. automobile vibration). While some vibrations may be regarded as useful and necessary, other vibrations can be suppressed or their energy can be converted into electrical energy by means of a harvester. For an instance, it can be used to vertically excite a parametric pendulum, which in turns rotates the pendulum and drives a generator to produce electrical energy. That form of vertical excitation can be chaotic or stochastic in the characteristic. Though both chaotic and stochastic excitations appear to be random and irregular, the chaotic excitation is deterministic while the stochastic excitation is characterized by using probability theory.

Various investigations have been performed on the dynamics of a parametric pendulum subjected to the stochastic excitation. For example, a stochastic forcing of Gaussian distribution was added to the forced pendulum model (Blackburn et al., 1995) and its effect in stabilizing and destabilizing the periodic motion of the parametric pendulum was studied by Blackburn (2006). Influence of the Wierner phase noise in the chaotic regime of a parametrically excited pendulum was numerically studied by Litak et al. (2008). A numerical study was conducted to investigate how stable rotational modes of a parametric pendulum were affected by introducing a stochastic forcing of Gaussian distribution by Horton and Wiercigroch (2008). Rotation of a parametric pendulum system subjected to an excitation with the Pierson-Moskowitz wave spectrum was studied by Najdecka (2013). Rotational motion of the Mathieu equation subjected to a narrow band excitation was investigated by Yurchenko et al. (2013). The stochastic response of a rotating pendulum mounted on a single degree-of-freedom (SDOF) base, which was subjected to narrow band excitation, was explored numerically by Yurchenko and Alevras (2013) and 
Alevras and Yurchenko (2014). Stochastic synchronization of rotating pendula mounted on a mutual elastic SDOF base subjected to narrow band excitation were numerically investigated by Alevras et al. (2014). Moreover, the parametric pendulum has been a subject of chaotic analysis as well. Influence of a nonlinear oscillator on an attached pendulum in the main parametric resonance region was studied by Warminski and Kecik (2006). The behavior of a parametric pendulum pivoted on a mass-spring-damper system was investigated analytically, numerically and experimentally around the principal resonance region by Warminski and Kecik (2009). The dynamics of an autoparametric pendulum-like system with a nonlinear suspension subjected to kinematic excitation was numerically and experimentally investigated by Kecik and Warminski (2011). The dynamics of a pendulum suspended on a forced Duffing oscillator was numerically explored by Brzeski et al. (2012).

As the rotational motion of parametric pendulum is advantageous for energy harvesting, it is desired to maintain that motion when it is subjected to any excitations. A few control strategies have been proposed for the purpose of sustaining rotational motion of the parametric pendulum. A time-delayed feedback method was employed to initiate and stabilize rotational motion of the pendulum by a feedback perturbation proportional to the difference between the present and a delayed state of the pendulum (Yukoi and Hikihara, 2011a). The tolerance of time-delayed feedback method with mistuned delay was experimentally investigated by Yukoi and Hikihara (2011b). The extended time-delayed feedback was proposed to maintain rotating solutions of the parametric pendulum to avoid bifurcations that destabilize the rotating orbit (De Paula et al., 2012). The robustness and sensitivity of the time-delayed feedback method with respect to varying excitation parameters and added noise was investigated by Vaziri et al. (2014). In addition, a velocity control method was proposed to effect a control torque about the angular axis if the angular velocity of the pendulum droped below a threshold (Najdecka, 2013). Alternative methods of control, such as a LQR method (Dadone et al., 2003) were deployed mainly for a pendulum-type system without continuous rotations.

The objective of this paper is to show an alternative mean of implementing an experimental stochastic process for facilitating investigations on the parametric pendulum for energy harvesting. In particular, the observation of rotational state of the pendulum under such an excitation is of interest in the current study. The proposed setup could exert a non-linear electromechanical force to the pendulum by means of an AC solenoid, which is powered by a solid-state-relay(SSR)coupled RLC circuit, and it was previously reported to exhibit period-1 rotations, period-1 oscillations and period-2 oscillations (Teh et al., 2015). The type of the parametric pendulum system considered belongs a special class of autoparametric systems; it has a mass-spring-damper system as the base and a pendulum is attached to it. Unlike the stochastic excitation of the parametric pendulum as the above-mentioned, the implementation of a stochastic excitation to the pendulum system in the current study is achieved by a random distribution of the input signal with respect to the duration of switching on and off the solenoid. In addition, a bang-bang control algorithm is tailored to address the problem of escaping the potential well when the noise is sufficiently destructive for positive energy generation. To implement the control algorithm and monitor potential energy generation, the experimental parametric system is enhanced with a control system by adding a rotational actuator, a generator and some necessary transducers. The main aim of this paper is to show that for stochastic energy sources commonly found in real life it is possible to introduce a minimal amount of the control input, while still harvesting more energy. This is an improvement to the previous work on periodic excitation by Teh et al. (2015).

The paper is organized in the following manner. The enhanced version of the experimental setup is first described in Section 2 together with its dynamic equations. The implementation of stochastic excitation for the experimental setup of the current study is described in Section 3. The bang-bang control algorithm that assists the pendulum motion is proposed in Section 4 . 
Some results on the pendulum system subjected to different levels of stochasticity are presented in Section 5. Finally, the paper is summarized and concluded in Section 6.

\section{Experimental setup}

The experimental setup of the parametric pendulum system given by Teh et al. (2015) has been extended to further investigation on rotations of the pendulum in the present work. The parametric pendulum system assembled for the experimental study on pendulum rotations under stochastic excitation is depicted in Fig. 1a. This configuration of the parametric pendulum system was previously described by Teh et al. (2015) regarding the functions and connections among the components. In the current study, the pendulum assembly has been modified by adding a L-bracket to mount the motor and the generator on to the pendulum assembly. The motor and generator are connected in parallel with the pendulum pivot by means of spur gears. The gear ratio among the pendulum pivot, motor and generator is 2:2:1. The overall assembly is suspended by means of a mechanical hook at the aluminium base plate.

(a)

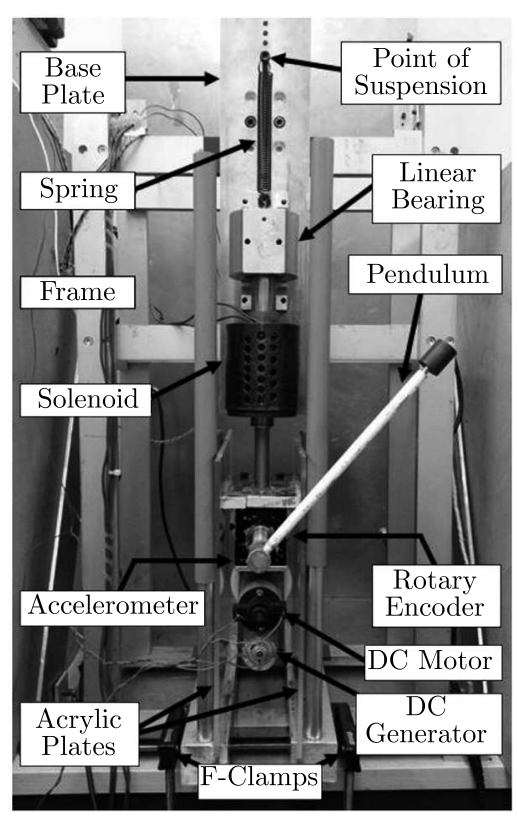

(b)

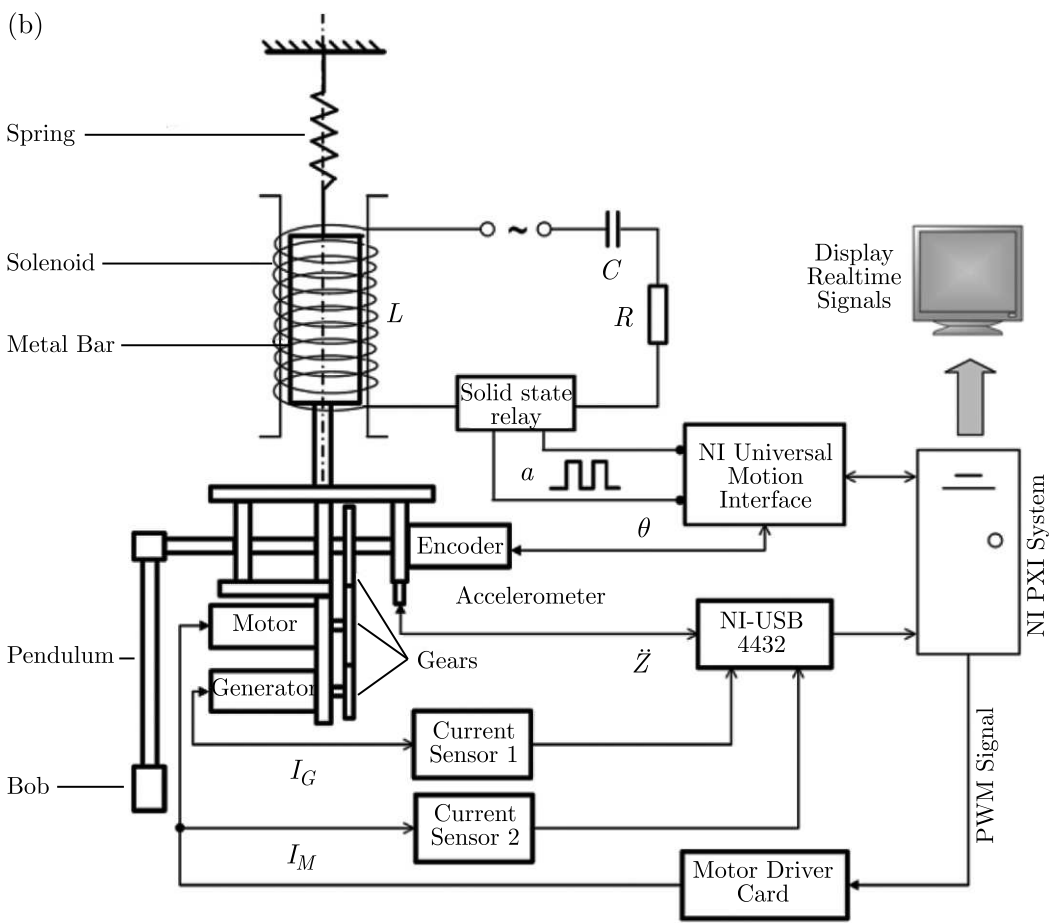

Fig. 1. (a) Experimental rig of the present work; (b) schematic of (a)

The schematic of the experimental setup is illustrated in Fig. 1b. The interface between the mechanical and electrical part of the system by Teh et al. (2015) has been enhanced in the present work to derive more information from the parametric pendulum setup while outputting commands simultaneously. Vertical oscillation of the pendulum assembly is achieved by exerting an electromagnetic force on the metal bar within the solenoid. The solenoid is connected in series with an external power supply and a capacitor to fulfill such forcing requirement, as depicted in Fig. 1b. It is effectively a RLC circuit and it is built in a similar way to Mendrela and Pudlowski (1992). A solid state relay is coupled with the RLC circuit to switch the solenoid on and off according to the input signal generated from the computer system. A non-linear electromechanical excitation of the pendulum assembly is generated by repeatedly switching the solenoid on and off, and a linear tension spring is used to complement the vertical oscillatory motion. 
Four transducers are employed to scavenge useful data from the experimental system. The angular displacement of the pendulum is measured using an Autonics incremental optical encoder (Model: E6B2-CWZ1X), and the signal is fed to monitoring system via National Instrument (NI) universal motion interface (Model: NI UMI-7774). Besides, the vertical motion of the pendulum assembly is measured using a Brüel \& Kjær piezoelectric accelerometer (Model: 4518-001). Two Allergo hall-effect based linear current sensors (Model: ACS711EX and ACS711LC) are used to respectively measure the electric current of the circuit of Crouzet DC motor (Model: 82800502) and Cytron DC generator (Model: SPG50-100K). The charge amplifier function of a NI data acquisition module (Model: NI USB-4432) is used to amplify the electrical signal generated by the accelerometer before being fed to the monitoring system. On the other hand, the analog voltage measurement function of the same data acquisition module is used to measure the voltage difference generated by the linear current sensors before being fed to the monitoring system.

The monitoring and logging of the experimental data is performed using NI PXI-embedded computer system (Model: NI PXI-8106). NI PXI-embedded computer system is also capable of simultaneously generating a signal for switching the solid state relay on-and-off and performing a control action on the pendulum axis based on the measured real-time data. LabVIEW is usedt o design a graphical user interface that could perform the above tasks based on NI PXI-embedded computer system. The NI motion controller module (Model: NI PXI-7350) of the computer system is used to generate a digital signal which is software-timed by a graphical user interface under a specific algorithm for switching the solid state relay on-and-off. Besides, the NI motion controller module is used to generate a hardware-timed pulse-width modulation (PWM) signal according to the computed control action, which is in the form of motor input voltage. The PWM signal is received by the Cytron DC motor driver card (Model: MD10C) and it draws electric current from a DC power supply to drive the DC motor according to the PWM signal.

In addition to the equations of motion for the mechanical system as well as governing equations describing the electrical circuit of the solenoid (Teh et al., 2015), the variation of current flowing through the DC motor are expressed as follows

$$
L_{M} \frac{d I_{M}}{d t}=U_{M}-R_{M} I_{M}-K_{M} \dot{\theta} \quad T_{M}=K_{M} I_{M}
$$

where $L_{M}$ is the motor inductance, $R_{M}$ is the motor resistance, $K_{M}$ is the motor torque constant, $U_{M}$ is the voltage applied to the motor armature, and $I_{M}$ is the motor current. Finally, the dynamics of the DC generator is expressed using the following equations

$$
L_{G} \frac{d I_{G}}{d t}=\eta K_{G} \dot{\theta}-R_{G} I_{G} \quad T_{G}=K_{G} I_{G}
$$

where $L_{G}$ is the motor inductance, $\eta$ is the gear ratio between the pendulum pivot and the generator, $R_{G}$ is the motor resistance, $K_{G}$ is the motor torque constant, and $I_{G}$ is the motor current.

\section{Stochastic excitation modeling}

Implementation of a stochastic excitation in the parametric pendulum system of this work is described in this Section. As aforementioned, the vertical excitation of the pendulum assembly is achieved by repeatedly switching the solenoid circuit on and off via a solid state relay. Variation of the timing for switching the solenoid on and off according to a random distribution could generate a stochastic excitation on the pendulum system. An example of the input signal of the solid state relay with varying on and off timing is shown in Fig. 2. The time interval between each peak of the input signal is different, and these values are generated from a random number generator. 
A frequency range from $1.2 \mathrm{~Hz}$ to $7.0 \mathrm{~Hz}$ is selected, in the first instance, as a basis for a random time period generator. The reciprocal of the upper and lower limit of frequency determines the corresponding limits of the time interval between peaks of the input signal. This frequency range has been selected such that these values can be implemented in the experiment such that the pendulum is rotated. This could maximize the transmission of vertical translational energy to the pendulum as it experiences an autoparametric resonance with the electromechanically-excited mass-spring-damper system.

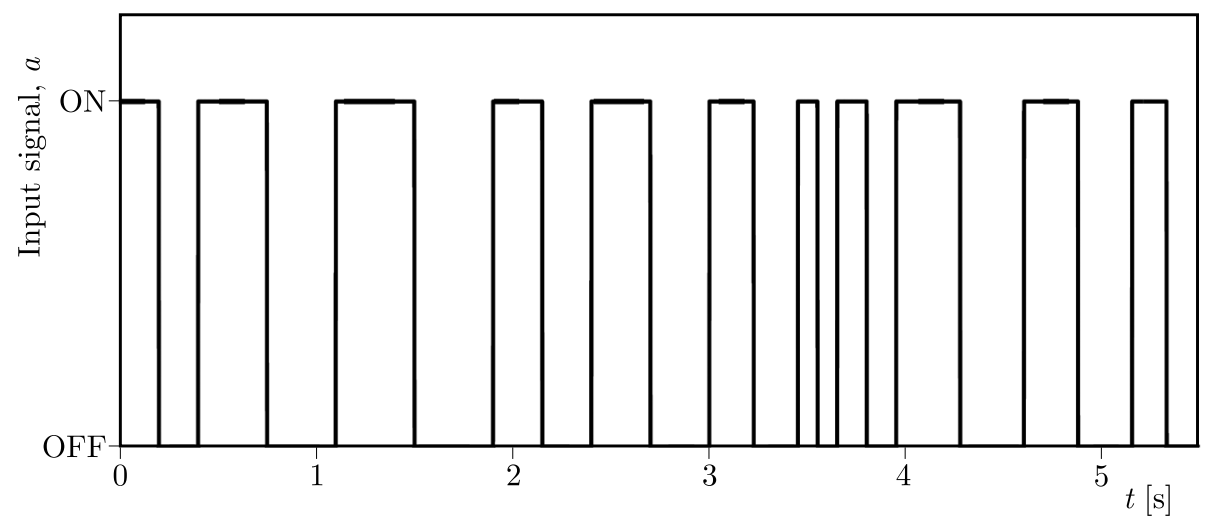

Fig. 2. Example of the input signal generated in a stochastic manner

The profile of the random distribution can be selected such that the random time period is generated according to either a set of discrete frequencies or a continuous variation of the frequency with respect to the frequency range of interest. Nonetheless, it is intended in this work to attempt emulating stochastic oscillatory motion of the pendulum assembly that could represent the sea wave excitation. The sea wave can be modeled as a stochastic process with a continuous function that can represent the state of the sea in the form of an energy spectrum; the wave spectrum. The Pierson-Moskowitz spectrum (Pierson and Moskowitz, 1964) is considered in the current study as the profile of the random distribution for the random number generator. It has been developed under the concept of a fully developed sea, and it is a one-parameter spectrum. The formulation of the Pierson-Moskowitz spectrum is given by

$$
S(\omega)=A \frac{g^{2}}{\omega^{5}} \exp \left(-\frac{16 \pi^{3}}{\omega^{4} T_{z}^{4}}\right) \quad T_{z}=B \sqrt{\frac{H_{s}}{g}}
$$

where $A=0.081, B=11.1, \omega$ is the frequency, and $H_{s}$ is the significant wave height which is defined as one third of the highest wave observed. From Eqs. (3.1), the profile of the wave spectrum depends solely on the parameter $H_{s}$. The same formulation was used by Najdecka (2013) to reconstruct the time history of the wave for stimulating the response of the pendulum system subjected to the sea wave excitation by means of an electro-dynamical shaker.

Figure 3a depicts the non-dimensionalized Pierson-Moskowitz spectrum.It is computed using Eqs. (3.1), and it features an asymmetric bell-shaped curve with a positive offset. It peaks at a nominal frequency, and that frequency can be expressed explicitly by equating to zero the derivative of $S(\omega)$ with respect to $\omega$, which yields the following expression

$$
\omega=\frac{2}{B} \sqrt{\frac{2 g}{H_{s}}} \sqrt[4]{\frac{\pi^{3}}{5}}
$$

Rearranging Eq. (3.2), one obtain an expression for $H_{s}$ in terms of other parameters

$$
H_{s}=\frac{8 \pi g}{\omega^{2} B^{2}} \sqrt{\frac{\pi}{5}}
$$


(a)

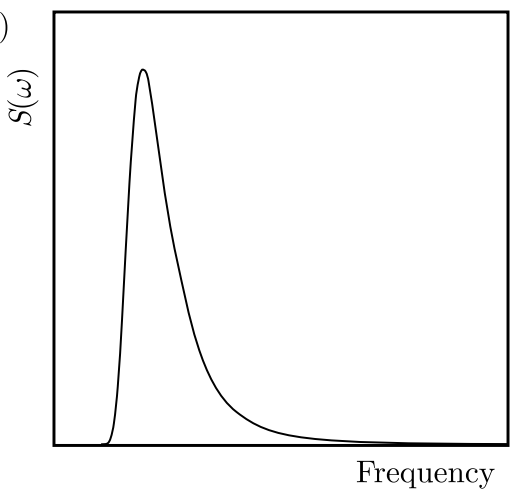

(b)

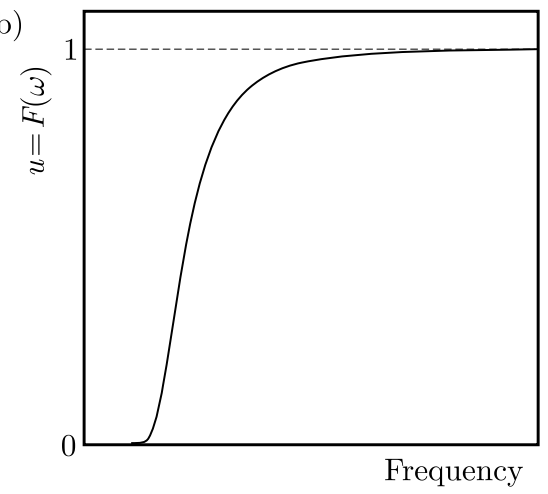

Fig. 3. Non-dimensionalized Pierson-Moskowitz wave spectrum; (b) cumulative distribution function of the Pierson-Moskowitz wave spectrum

The value of the parameter $H_{s}$ can thus be determined in order to match the nominal frequency of the wave spectrum as close as possible to the operating frequency of the pendulum system.

Generation of a random time period based on the Pierson-Moskowitz spectrum is performed by using the inverse transform technique in this work. The inverse transform technique, also known as the Smirnov transform, is useful for generating random variates with an arbitrary continuous distribution function (Devroye, 1986). To utilize this technique, the selected random distribution is first integrated and then rescaled to obtain its cumulative distribution function $F$, such that it maps a number in the domain to a probability between $[0,1]$, as illustrated in Fig. 3b. This technique works as follows. A random number $u$ is generated from the standard uniform distribution in the interval of $[0,1]$. The generated $u$ is then interpolated with the rescaled cumulative distribution function to locate the frequency $\omega$. In other words, the inverse function of $F$ is sought to determine the frequency $\omega$ that corresponds to the generated random number $u$, with their relationship being expressed as follows

$$
\omega=F^{-1}(u) \quad \Delta t=\frac{2 \pi}{\omega}-T_{0} \quad T_{n e w}=T_{0}+C_{1} \Delta t
$$

In Eq. $(3.4)_{2}$, the random time period is decomposed into two components, namely the nominal time period $T_{0}$ and the offset time period introduced by the wave spectrum $\Delta t . T_{0}$ is also the inverse of the nominal frequency of the wave spectrum. Finally, a factor within the range of $[0,1] C_{1}$ is introduced to control the stochasticity of the random time period in Eq. $(3.4)_{3}$, which yields the new time period for that cycle.

To generate a train of square pulses with different timing for switching the solenoid on and off in the experiment continuously, the above procedure can be repeated in a timer loop. For each iteration, the timer will start counting once a new time period is generated, and a square wave and the command signal will be computed based on that time period. The command signal generated by the computer system can be written as

$$
a= \begin{cases}1 & \text { if } t\left(\bmod T_{\text {new }}\right)<\frac{1}{2} T_{\text {new }} \\ 0 & \text { otherwise }\end{cases}
$$

where the units of the timer time $t$, the new time period $T_{n e w}$ is in seconds. By using the equations above, the generated input signal will be a train of square pulses with a fixed duty cycle of $50 \%$. The timer will be reset automatically once the timer counts until $T_{n e w}$. The time period for each iteration is then different, and so the on-and-off timing for each square wave in the pulse train, which is illustrated in Fig. 2. LabVIEW environment is used to program the above algorithm in this work. 
Figure 4a depicts the time history of the vertical displacement of the pendulum support generated by the proposed stochastic excitation. It is observed at a root-mean-squared variac voltage of $110 \mathrm{~V}_{\mathrm{rms}}$. The nominal frequency of the spectrum is set to be $1.9 \mathrm{~Hz}$, such that the corresponding value of $H_{s}$ is $0.011 \mathrm{~m}$. The pendulum is constrained at its downright position during the measurement, and $C_{1}$ is set to 1 to utilize the full scale of the Pierson-Moskowitz spectrum. From Fig. 4a, the amplitude of oscillation and time interval between two peaks is observed to be varying with time, which suggests that an irregular oscillation of the massspring-damper system could be generated by using the proposed stochastic excitation. The FFT spectrum of the time history (line 1) in Fig. 4b suggests that the vertical oscillation is stochastic in nature. The energy content of the vertical oscillation lies within a range of $1.2 \mathrm{~Hz}$ to $6 \mathrm{~Hz}$, and it also resembles a bell-shaped curve with a positive offset. The spectrum is smoothened (line 2) and it displays a major peak in close vicinity of the nominal frequency of the PiersonMoskowitz spectrum. By comparing it with the Pierson-Moskowitz spectrum (line 3), it can be seen that the smoothened spectrum of the time history is in good correspondence with the desired power density profile with mild slight discrepancy between the shapes of the spectra, which is representative of many vibratory systems. The above observations show the viability of the excitation mechanism in the current study to attempt emulating a stochastic excitation on a solenoid-powered mass-spring-damper system.

(a)

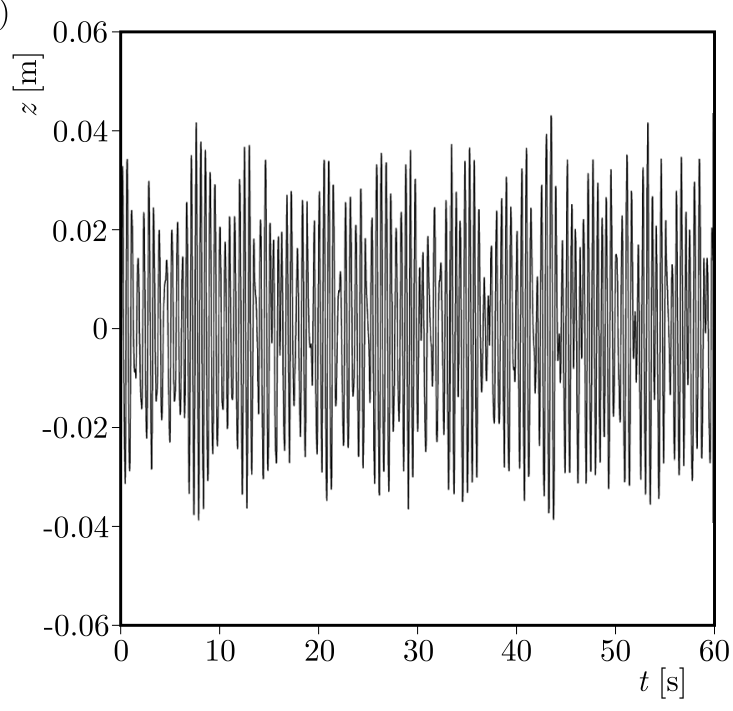

(b)

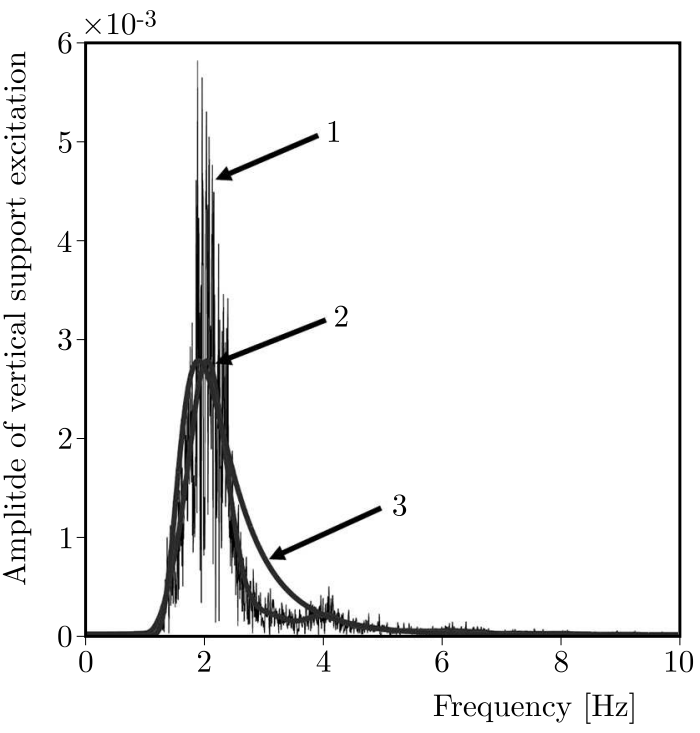

Fig. 4. (a) Time history of the vertical support excitation using the proposed stochastic excitation for $V=130 \mathrm{~V}_{\mathrm{rms}}$ and $\omega_{0}=1.9 \mathrm{~Hz}$; (b) FFT spectrum of (a) compared with the Pierson-Moskowitz wave spectrum generated for $H_{s}=0.011 \mathrm{~m}$

\section{Rotation control of pendulum}

A bang-bang control is suggested in the current study to promote rotational motion of the pendulum as it is subjected to a random excitation. The objective of control is to assist motion of the pendulum despite randomness of the vertical excitations. The nature of the control strategy is intuitive; while the pendulum is rotating, a control torque will be exerted along the rotational direction if the control system senses that the pendulum is experiencing a destructive coupling torque arisen from the pendulum support that opposes the current motion of pendulum. The real-time information of the angular displacement and velocity of the pendulum and the vertical acceleration of the pendulum support are assigned as the feedback signal of the controller and some conditions are defined based on those signals to exert control action. The function of each 
signal is detailed as follows. The signal of angular displacement of the pendulum indicates the position of the pendulum from its downright equilibrium position. On the other hand, the signal of angular velocity of the pendulum indicates the direction and the rate at which the pendulum moves. Lastly, the signal of vertical acceleration of the pendulum support indicates the direction of the summation of forces acting on the pendulum support.

The rotating plane of the pendulum splits up into two halves, namely left-half plane and right-half plane, and they form control envelopes. The conditions for activating the control action is different for each plane. For the left-half plane (i.e. $2 n \pi<\theta<(2 n+1) \pi, n \in N)$

$$
U_{M}(t)= \begin{cases}k_{1}+k_{2} \sin \theta & \text { if } \ddot{z}>0 \wedge 0 \leqslant \dot{\theta}<\dot{\theta}_{\text {min }} \\ -k_{3} & \text { if } \ddot{z}<0 \wedge-\dot{\theta}_{\text {min }}<\dot{\theta}<0 \\ 0 & \text { otherwise }\end{cases}
$$

where $U_{M}$ is the input to the DC motor, $k_{1}$ and $k_{2}$ are control parameters applied when the pendulum is climbing from its downright equilibrium, and $k_{3}$ is the control parameter applied when the pendulum is falling from its upright equilibrium position. Suppose that the pendulum is rotating in the left-half plane. The coupling torque will be destructive in that plane if the pendulum support is suddenly accelerated upwards while the pendulum is rotating in the clockwise direction, and this may cause the pendulum to lose angular velocity and fall to its downright equilibrium position. Hence, a control torque will be exerted along the clockwise direction if the pendulum support is accelerated upwards and the rate at which the pendulum is rotating is lower than the threshold angular velocity $\dot{\theta}_{\text {min }}$, as stated in the first condition of Eq.(4.1). A sinusoidal term is added to compensate for the torque due to gravity while the pendulum is climbing from its downright equilibrium position. On the other hand, the coupling torque will also be destructive in the left-half plane if the pendulum support is suddenly accelerated downwards while the pendulum is rotating in the counter-clockwise direction. A control torque of the counter-clockwise direction will then be exerted if the pendulum support is accelerated downwards and the rate at which the pendulum is rotating is greater than the negative threshold angular velocity, as stated in the second condition of Eq. (4.1). The control action will otherwise not be exerted in the left-half plane if the above conditions are not met. Likewise, for the right-half plane (i.e. $(2 n+1) \pi<\theta<2(n+1) \pi, n \in N)$

$$
U_{M}(t)= \begin{cases}-\left(k_{1}+k_{2} \sin \theta\right) & \text { if } \ddot{z}>0 \wedge-\dot{\theta}_{\text {min }}<\dot{\theta}<0 \\ k_{3} & \text { if } \ddot{z}<0 \wedge 0 \leqslant \dot{\theta}<\dot{\theta}_{\text {min }} \\ 0 & \text { otherwise }\end{cases}
$$

Due to an inequality condition at $\dot{\theta}=0$, the control algorithm is also capable of initiating pendulum motion regardless of the initial conditions besides maintaining the rotation state of the pendulum, which is to be shown in Section 5 .

\section{Pendulum rotation subjected to the proposed stochastic excitation}

The experimental rig, as described in Section 2, is used to implement the proposed method of varying the on-and-off timing of the solenoid to induce stochastic excitations on the pendulum system. The pendulum system is tested at different levels of stochasticity by varying the onand-off timing, and the observation of rotational state of pendulum under such excitation is particularly of interest in view of energy scavenging. The control algorithm, as described in Section 4, is used to drive the rotational actuator in attempting to assist the rotation of the pendulum when it could not be sustained. 
An AC supply voltage of $130 \mathrm{~V}_{\mathrm{rms}}$ and a nominal frequency of $1.9 \mathrm{~Hz}$ is chosen to generate experimental results in the current study. The selected parameters lie within the resonance area of the pendulum system, which is advantageous for generating rotation of the pendulum. The pendulum system is first excited by switching the RLC circuit of the solenoid on-and-off with a regular time interval. The control algorithm is then activated and the pendulum is actuated from its downright equilibrium position to yield period-1 rotation. A buffer period of approximately $30 \mathrm{~s}$ is required to let the pendulum motion settle to period- 1 rotation. After that, stochasticity is introduced to the excitation and the level of noise is controlled by manipulating $C_{1}$ in Eq. $(3.4)_{3}$. The pendulum system is run for a period of $300 \mathrm{~s}$, and useful experimental data is logged simultaneously. As the rotational process is stochastic, the measurement for the same noise level is to be repeated for another 9 sets to obtain the average accumulated net energy. The control parameters $k_{1}, k_{2}, k_{3}$ and $\dot{\theta}_{\text {min }}$ are experimentally optimized and fixed at 8, 4, 1 and 7.5, respectively, for all noise levels throughout the experiment.

(a)
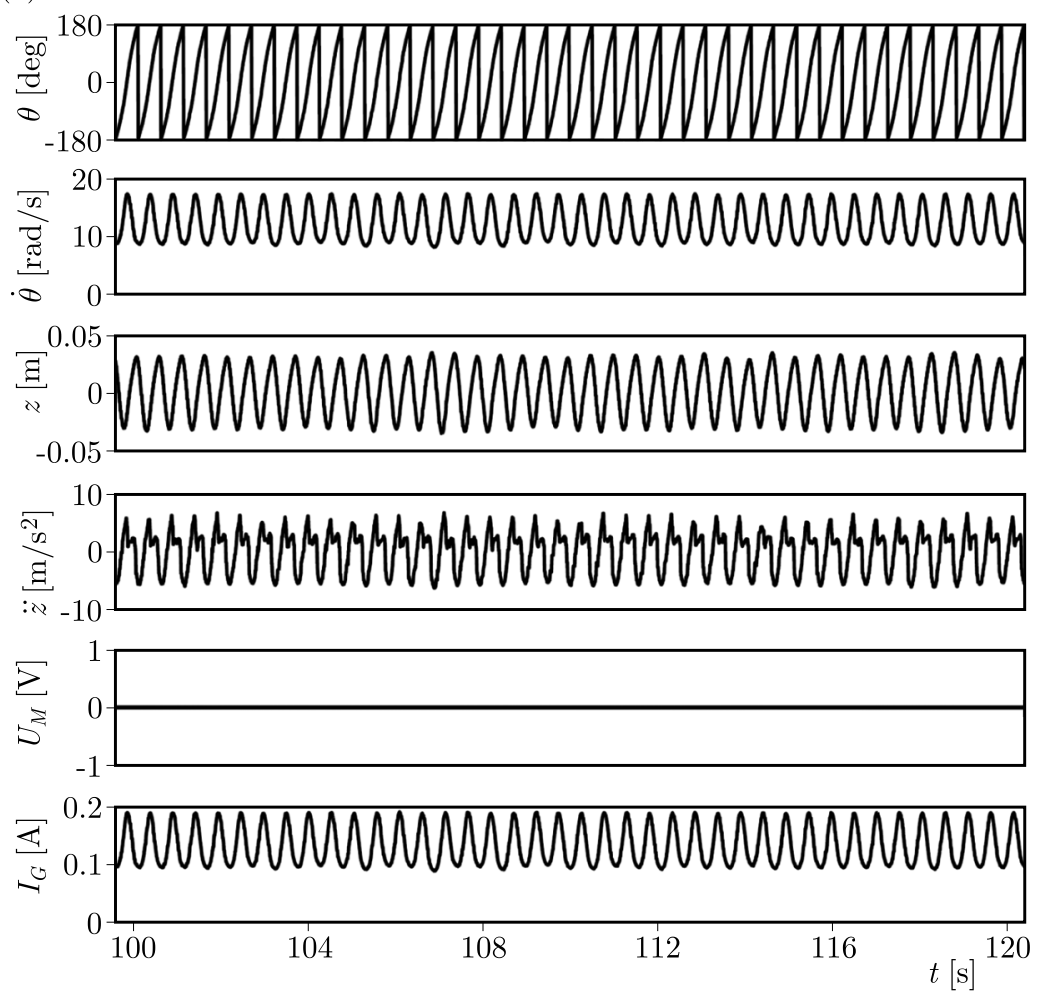

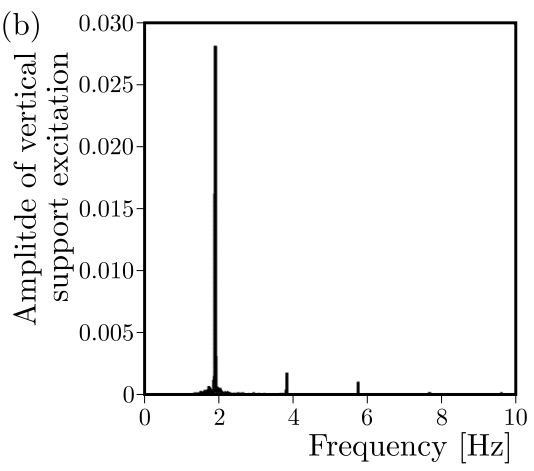

(c)

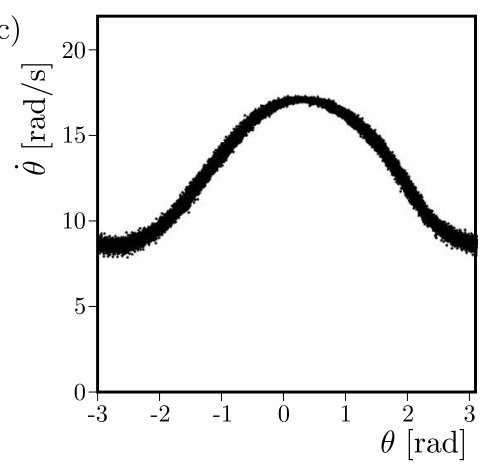

Fig. 5. (a) Time response of the pendulum system for $C_{1}=0$; (b) FFT spectrum for vertical support excitation generated using experimental results; (c) phase portrait of the pendulum axis

Figure 5a demonstrates a sample time history of the pendulum system when $C_{1}=0$ for a period of $21 \mathrm{~s}$. In this case, the noise is disabled in the timer loop and the solenoid is switched on and off in a regular time interval, which is $0.526 \mathrm{~s}$. Thus, the pendulum exhibits period- 1 rotation as it experiences an autoparametric resonance with the vertical support excitation, which is also periodic, after it is actuated from its rest position. The quality of the periodic excitation can be assessed via its FFT spectrum, which, see Fig. 5b, shows a considerably discrete spectrum with a major peak at the fundamental frequency and subsequently minor peaks at its integer multiple with reducing the magnitude. On the other hand, it can be seen from the phase portrait of the pendulum axis in Fig. 5c that the pendulum rotates with fluctuating angular velocity, which is bounded within the range of $[7.9,17.2] \mathrm{rad} / \mathrm{s}$, under the regular excitation from the pendulum support. As the source of energy to maintain stable rotation is solely from the vertical support 
excitation, no control action is required from the DC motor. The power of the generator will thus be the only contributor to the accumulated net energy at the end of the measurement, which serves as a benchmark for comparison with the accumulated net energy at higher noise levels.

The period- 1 rotation of the pendulum system ceases to be stable when $C_{1}$ is increased up to 0.04 . Hence, control assistance is necessary when $C_{1}$ is set to be within the range of $(0.04,1]$ in order to maintain the rotational state of the pendulum. In the next case, the sample of the time history of the pendulum system when it is subjected to stochastic excitation in a moderate scale $\left(C_{1}=0.3\right)$ is illustrated in Fig. 6a. The time interval that the solenoid is switched on-and-off is varied, in this case, within the range of $[0.411,0.617] \mathrm{s}$. Period-1 rotation could still be observed most of the time, as the occurrence of the moments when the vertical support excitation is less destructive. Consequently, less control effort is required in this case with only four instances of the control voltage input observed in this interval of the experimental data. FFT spectrum of the vertical support excitation in Fig. $6 \mathrm{~b}$ reveals that the spectrum is no longer discrete, and it is stochastic in nature. The amplitude of the vertical excitation at the fundamental frequency becomes one-fourth of its periodic counterpart. The energy is slightly spread in the vicinity of the fundamental frequency as a consequence of slight variation in the on-and-off time interval. The shape of the spectrum, on the other hand, is somewhat similar to that of the PiersonMoskowitz wave spectrum with a narrower and sharper peak, as illustrated by the smoothened curve (line 2). The angular velocity at which the pendulum rotates, fluctuates within the range of $[4.8,19.4] \mathrm{rad} / \mathrm{s}$ as depicted in Fig. $6 \mathrm{c}$. It is wider than its periodic counterpart. The occasional randomness of the excitation causes the pendulum rotation to lose angular velocity at some instances, which is then rectified by the control action, and hence the lower limit of angular velocity range is extended when stochasticity is introduced to the excitation.

(a)
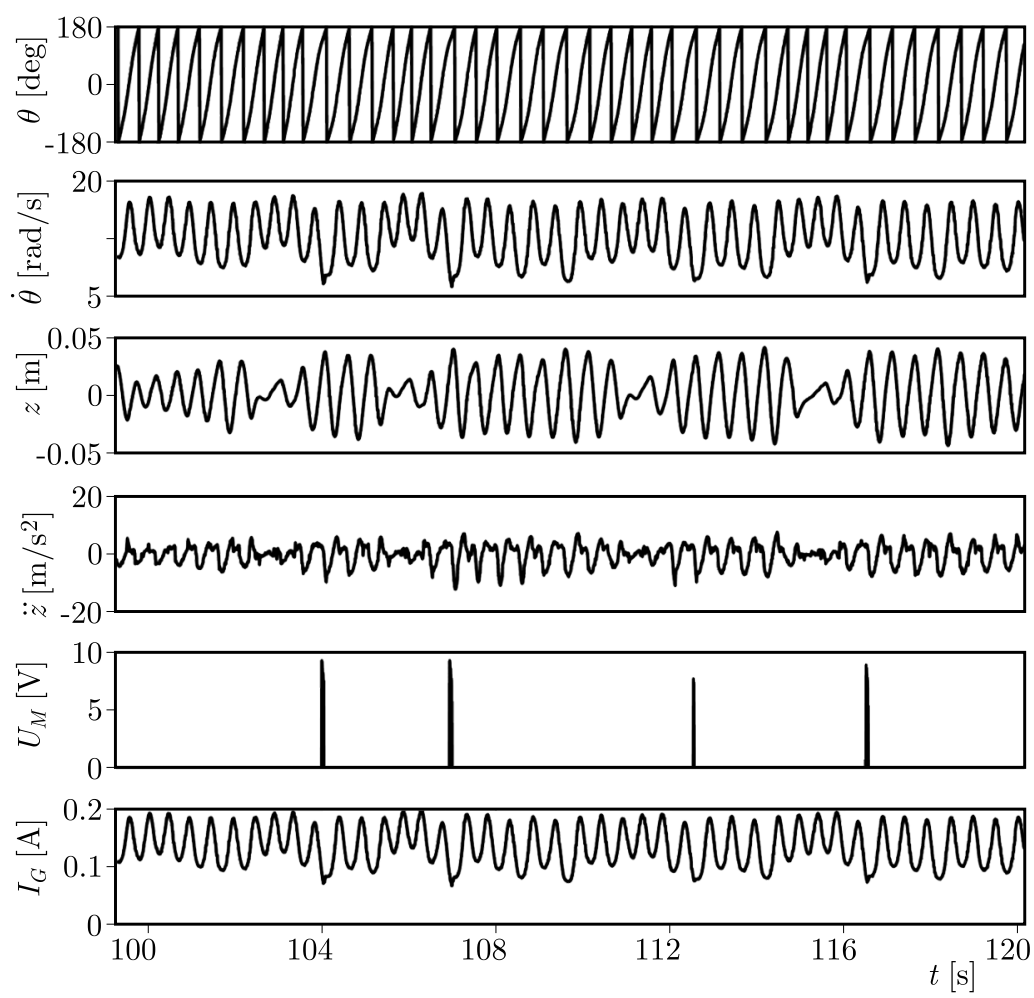

(b)

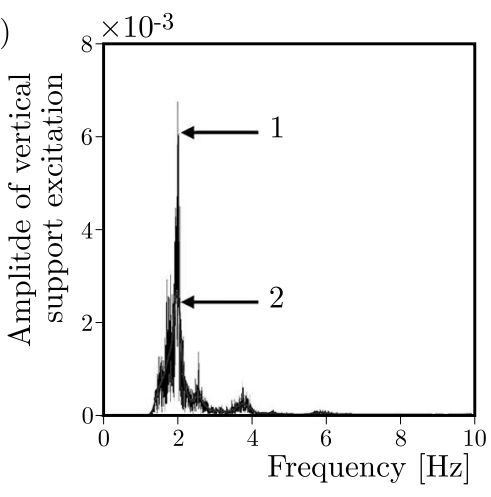

(c)

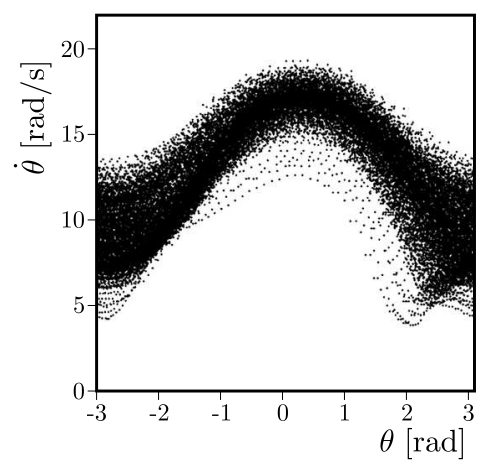

Fig. 6. (a) Time response of the pendulum system for $C_{1}=0.3$; (b) FFT spectrum for vertical support excitation generated using experimental results; (c) phase portrait of the pendulum axis 
The sample time history of the pendulum system when $C_{1}=1$ is illustratedin Fig. 7a. In this case, the full scale of the Pierson-Moskowitz spectrum is used, and the time interval that the solenoid is switched on-and-off varies within the range of $[0.143,0.830] \mathrm{s}$. It can be observed from the vertical axis data that the vertical support excitation is irregular at higher level of noise, which causes the pendulum trajectory to be intermittently distracted from its rotation attractor. This necessitates frequent control actions to assist the pendulum in regaining angular momentum and synchronizing again with the vertical support excitation. Accordingly, more control efforts are required at a higher level of noise, which is seen in the fifth row of Fig. 7a with 15 instances of the control voltage input observed in this interval of the experimental data. The contents of irregularities can be assessed via the FFT spectrum of the vertical support excitation in Fig. 7b. The smoothened spectrum (line 2) depicts a curve, and the shape of the spectrum is similar to that displayed in Fig. 3a. The spectrum peaks at the fundamental frequency, and the amplitude of the vertical support excitation at the fundamental frequency is lesser than in the previous cases. This is expected as the energy of the vertical excitation has been spread over a wider frequency range due to the maximum difference of $0.687 \mathrm{~s}$ in varying the time interval. The vertical support excitation does not possess enough energy in the vicinity of the fundamental frequency to sustain the pendulum rotation. This prompts the pendulum to lose its angular velocity and almost stall at some point in time before the controller is alerted to react to sudden disturbances. This is seen in the phase portrait of the pendulum axis, where the fluctuation range of angular velocity is widened to $[3.6,19.8] \mathrm{rad} / \mathrm{s}$.

$$
E_{\text {accumulated }}=\eta K_{G} \int_{t_{1}}^{t_{2}} I_{G} \dot{\theta} d t-K_{M} \int_{t_{1}}^{t_{2}} I_{M} \dot{\theta} d t
$$

(a)
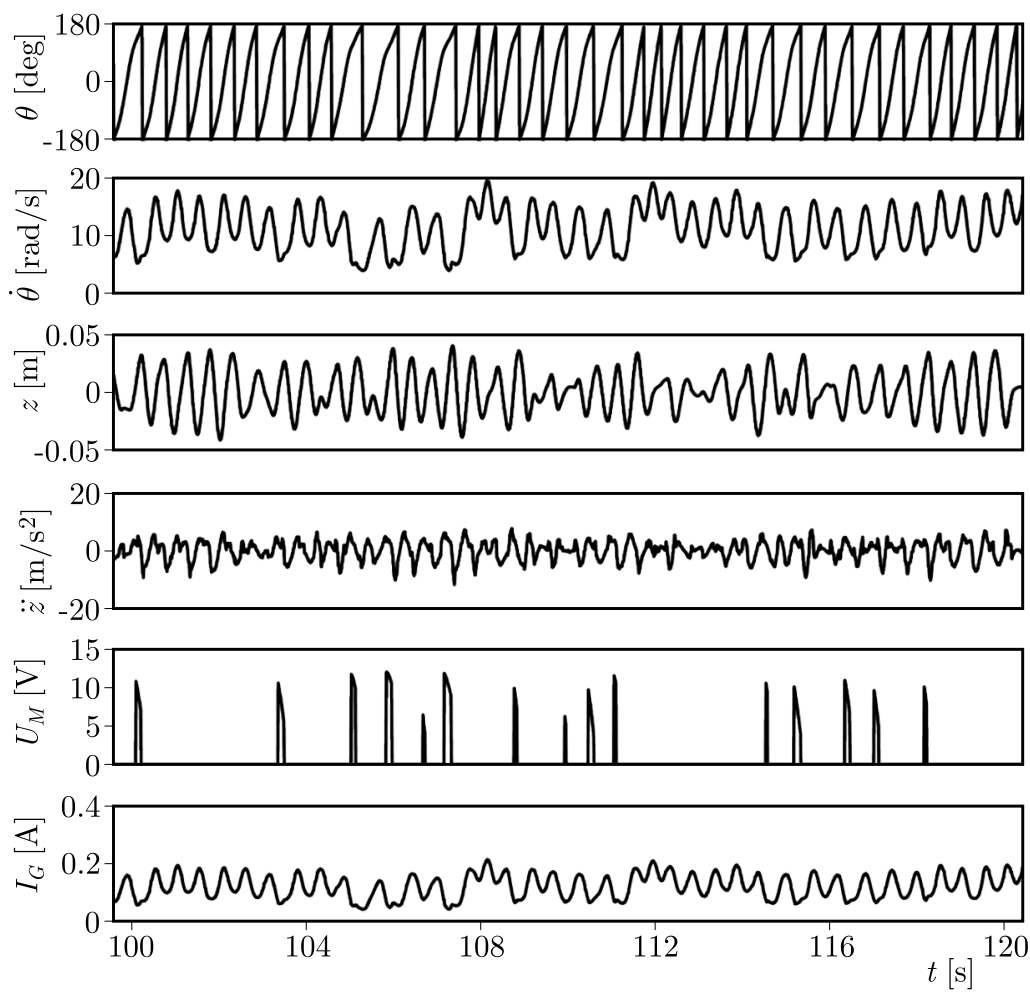

(b)

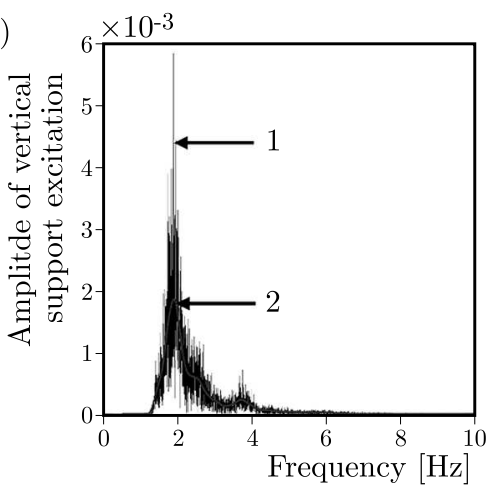

(c)

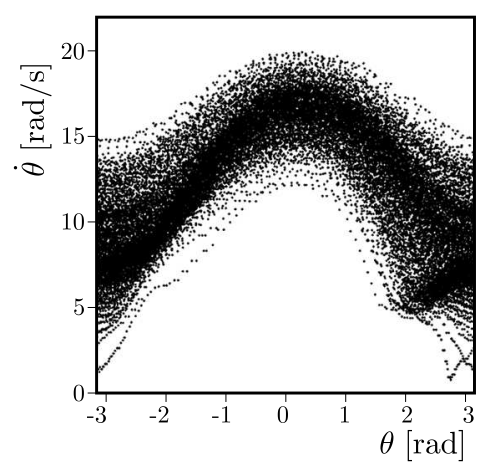

Fig. 7. (a) Time response of the pendulum system for $C_{1}=1$; (b) FFT spectrum for vertical support excitation generated using experimental results; (c) phase portrait of the pendulum axis 
Energy generation is measured via current measurement from the generator, and the accumulated net energy is computed using Eq. (5.1) in real time during the course of the experiment in all the above cases. In Eq. (5.1), $t_{1}$ is the time when computation of the accumulated net energy begins and $t_{2}$ is the time when computation of the accumulated net energy ends. Figure 8 illustrates sample time histories of the accumulated net energy for the above three cases computed over a period of $t_{2}-t_{1}=300 \mathrm{~s}$. In the case when the noise is disabled $\left(C_{1}=0\right)$, pendulum rotation is sustainable without interference from the controller. Hence, the power generated from the DC generator is the sole contributor to computation of the accumulated net energy, and it can be seen from Fig. 8 that the accumulated net energy for $C_{1}=0$ increases steadily as the experiment progresses. The average accumulated net energy at the end of $300 \mathrm{~s}$, obtained using 10 sets of data, is $24.51 \mathrm{~J}$ for $C_{1}=0$. On the other hand, in the cases when noise is added to the vertical excitation, control effort is required to sustain the pendulum rotation for maximizing the energy output from the generator. Therefore, energy is invested for exerting the control effort by means of actuation of the DC motor and this is taken into consideration while computing the accumulated net energy. For the case when a moderate level of noise is introduced to the excitation $\left(C_{1}=0.3\right)$, occasional control torque is required to maintain the pendulum rotation and some energy is invested to achieve this purpose. The average accumulated net energy at the end of $300 \mathrm{~s}$ is $14.01 \mathrm{~J}$ for $C_{1}=0.3$, which is $57.16 \%$ of its periodic counterpart. For the case when the full scale of Pierson-Moskowitz spectrum is introduced to the excitation $\left(C_{1}=1\right)$, the accumulated net energy is observed to be the least. Its average accumulated net energy at the end of $300 \mathrm{~s}$ is calculated as $4.67 \mathrm{~J}$, which is $19.05 \%$ of the case when $C_{1}=0$. This is expected as more control input is necessary to prevent the pendulum motion from losing angular velocity when the pendulum is frequently subjected to the destructive vertical excitation.

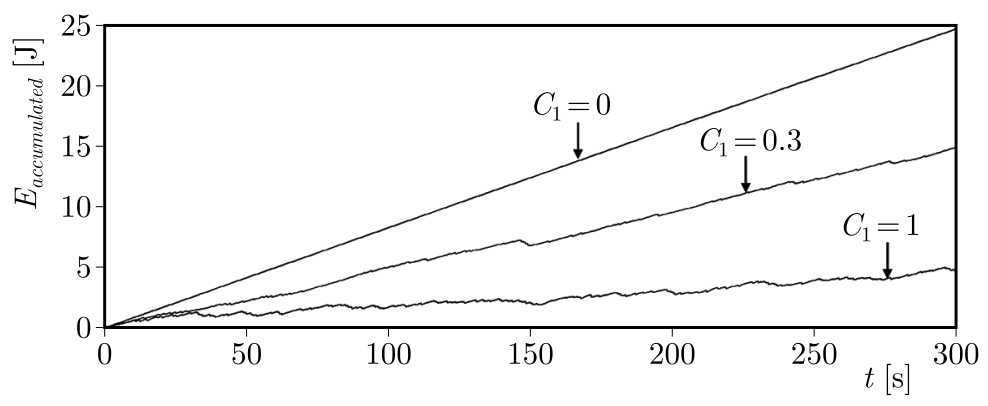

Fig. 8. Comparison of time histories of accumulated net energy for $C_{1}=0, C_{1}=0.3$ and $C_{1}=1$

\section{Conclusion}

An experimental pendulum system, which exerts a non-linear vertical excitation on a pendulum via a RLC-circuit powered solenoid is used to conduct investigations on pendulum rotation subjected to a stochastic excitation. The pendulum system, which was previously reported in the open literature, is enhanced with a control system by adding a rotational actuator and some necessary transducers. In addition, a generator is mounted in parallel to the pendulum axis for extracting energy out of rotating motion of the pendulum.

The stochastic excitation of the pendulum system is accomplished by a variable time interval for switching the RLC-circuit on and off according to a random distribution. In particular, the Pierson-Moskowitz wave spectrum is selected as the random distribution, and the generation of the random time interval is implemented in the experiment using an inverse transform technique. On the other hand, a bang-bang control algorithm is tailored to assist rotational motion of the pendulum as it is subjected to the random excitation. The main inputs of the controller are 
the displacement and velocity of the angular axis and the acceleration of the vertical axis. The output is the command signal to the rotational actuator.

Using the proposed method of stochastic excitation and control scheme, the experimental results are illustrated for various levels of noise introduced to the vertical support excitation. The observations demonstrate the viability of the pendulum system in emulating a random excitation, which is similar to that of the sea wave excitation in experimental investigations of a vertical pendulum. The enhanced version of the experiment apparatus takes advantage of the rotational actuator and generator to respectively assist the pendulum motion and benefit from motion itself when it is excited vertically. In addition, the proposed control scheme can effectively retain the rotational state of the pendulum for all the above cases without suffering an energy deficit. The energy cost of the control increases with the level of noise introduced to the vertical excitation.

\section{References}

1. Alevras P., Yurchenko D., 2014, Stochastic rotational response of a parametric pendulum coupled with an SDOF system, Probabilistic Engineering Mechanics, 37, 124-131

2. Alevras P., Yurchenko D., Naess A., 2014, Stochastic synchronization of rotating parametric pendulums, Meccanica, 49, 8, 1945-1954

3. Blackburn J.A., 2006, Noise activated transitions among periodic states of a pendulum with a vertically oscillating pivot, mediated by a chaotic attractor, Proceedings of the Royal Society A: Mathematical, Physical and Engineering Science, 462, 2067, 1043-1052

4. Blackburn J.A., Grønbech-Jensen N., Smith H.J.T., 1995, Stochastic noise and chaotic transients, Physical Review Letters, 74, 6, 908

5. Brzeski P., Perlikowski P., Yanchuk S., Kapitaniak T., 2012, The dynamics of the pendulum suspended on the forced Duffing oscillator, Journal of Sound and Vibration, 331, 24, 5347-5357

6. Dadone P., Lacarbonara W., Nayfeh A.H., Vanlandingham H.F., 2003, Payload pendulation reduction using a variable-geometry-truss architecture with LQR and fuzzy controls, Journal of Vibration and Control, 9, 805-837

7. De Paula A.S., Savi M.A., Wiercigroch M., Pavlovskaia E., 2012, Bifurcation control of a parametric pendulum, International Journal of Bifurcation and Chaos, 22, 1250111

8. Devroye L., 1986, Non-uniform Random Variate Generation, Springer-Verlag, New York

9. Horton B.W., Wiercigroch M., 2008, Effects of heave excitation on rotations of a pendulum for wave energy extraction, IUTAM Symposium on Fluid-Structure Interaction in Ocean Engineering, $117-128$

10. KeciK K., Warminski J., 2011, Dynamics of an autoparametric pendulum-like system with a nonlinear semiactive suspension, Mathematical Problems in Engineering

11. Litak G., Borowiec M., Wiercigroch M., 2008, Phase locking and rotational motion of a parametric pendulum in noisy and chaotic conditions, Dynamical Systems, 23, 3, 259-265

12. Mendrela E.A., Pudlowski Z.J., 1992, Transients and dynamics in a linear reluctance selfoscillating motor, IEEE Transactions on Energy Conversion, 7, 1, 183-191

13. NAJDECKA A., 2013, Rotating dynamics of pendula systems for energy harvesting from ambient vibrations, Ph.D. Thesis, University of Aberdeen, Scotland

14. Pierson W.J., Moskowitz L., 1964, A proposed spectral form for fully developed wind seas based on the similarity theory of SA Kitaigorodskii, Journal of Geophysical Research, 69, 24, 5181-5190

15. Teh S.-H., Chan K.-H., Woo K.-C., Demrdash H., 2015, Rotating a pendulum with an electromechanical excitation, International Journal of Non-Linear Mechanics, 70, 73-83 
16. VAziri V., Najdecka A., Wiercigroch M., 2014, Experimental control for initiating and maintaining rotation of parametric pendulum, The European Physical Journal Special Topics, 223, 795-812

17. WARminski J., KeCiK K., 2006, Autoparametric vibrations of a nonlinear system with pendulum, Mathematical Problems in Engineering

18. WARMinski J., KECIK K., 2009, Instabilities in the main parametric resonance area of a mechanical system with a pendulum, Journal of Sound and Vibration, 322, 3, 612-628

19. Yukoi Y., Hikihara T., 2011a, Start control of parametric pendulum into periodic rotation, Transactions of the Institute of Systems, Control and Information Engineers, 24, 54-60

20. Yukoi Y., Hikihara T., 2011b, Tolerance of start-up control of rotation in parametric pendulum by delayed feedback, Physics Letters A, 375, 1779-1783

21. Yurchenko D., Alevras P., 2013, Stochastic dynamics of a parametrically base excited rotating pendulum, Procedia IUTAM, 6, 160-168

22. Yurchenko D., Naess A., Alevras P., 2013, Pendulum's rotational motion governed by a stochastic Mathieu equation, Probabilistic Engineering Mechanics, 31, 12-18

Manuscript received March 14, 2015; accepted for print October 26, 2015 